\begin{tabular}{|c|l|}
\hline Title & UItraslow dielectric relaxation process in supercooled polyhydric alcohols \\
\hline Author(s) & Yomogida, Y oshiki; Minoguchi, A yumi; Nozaki, Ryusuke \\
\hline Citation & $\begin{array}{l}\text { Physical review E, 73(4), 041510-1-041510-6 } \\
\text { https://doi.org/L0.1103/PhysRevE.73.041510 }\end{array}$ \\
\hline Issue Date & 2006-04 \\
\hline Doc URL & http://hdl.handle.net/2115/9101 \\
\hline Rights & Copyright $\odot 2006$ A merican Physical Society \\
\hline Type & article \\
\hline File Information & PhysRevE_v73a041510.pdf \\
\hline
\end{tabular}

Instructions for use 


\title{
Ultraslow dielectric relaxation process in supercooled polyhydric alcohols
}

\author{
Yoshiki Yomogida, Ayumi Minoguchi, and Ryusuke Nozaki \\ Division of Physics, Graduate School of Science, Hokkaido University, Sapporo 060-0810, Japan \\ (Received 24 October 2005; revised manuscript received 27 February 2006; published 19 April 2006)
}

\begin{abstract}
Complex permittivity was obtained on glycerol, xylitol, sorbitol and sorbitol-xylitol mixtures in the supercooled liquid state in the frequency range between $10 \mu \mathrm{Hz}$ and $500 \mathrm{MHz}$ at temperatures near and above the glass transition temperature. For all the materials, a dielectric relaxation process was observed in addition to the well-known structural $\alpha$ and Johari-Goldstein $\beta$ relaxation process [G. P. Johari and M. Goldstein, J. Chem. Phys. 53, 2372 (1970)]. The relaxation time for the new process is always larger than that for the $\alpha$ process. The relaxation time shows non-Arrhenius temperature dependence with correlation to the behavior of the $\alpha$ process and it depends on the molecular size systematically. The dielectric relaxation strength for the new process shows the effect of thermal history and decreases exponentially with time at a constant temperature. It can be considered that a nonequilibrium dynamics causes the new process.
\end{abstract}

DOI: 10.1103/PhysRevE.73.041510

PACS number(s): 64.70.Pf, 61.25.Em, 77.22.Gm

\section{INTRODUCTION}

One of the interesting features of supercooled liquids is coexistence of many dynamic processes with different characteristic times. Recent studies by means of broadband dielectric spectroscopy have revealed that there are at least four processes commonly recognized at a temperature near the glass transition temperature $T_{g}[1]$. These are the $\alpha$ process, the slow $\beta$ process (so-called Johari-Goldstein process [2]), the fast $\beta$ process and the boson peak. The $\alpha$ process has the largest relaxation time and is considered to be the structural relaxation process directly connected to the glass transition phenomena. The slow $\beta$ process shows up at a temperature where the $\alpha$ process starts to follow a nonArrhenius manner with decreasing temperature. Recent investigations on polyhydric alcohols (sorbitol, xylitol, glycerol) by means of the broadband dielectric spectroscopy have suggested that the slow $\beta$ process is concerned with local fluctuations among molecules [3-6]. The relaxation times of these two processes have strong temperature dependence, especially for the non-Arrhenius $\alpha$ process. On the other hand, the fast $\beta$ process and the boson peak show essentially no temperature dependence and the relaxation times are very small. It is considered that the fast $\beta$ process is connected to the caged dynamics and the boson peak is caused by local librations of the molecules and/or atomic groups. These dielectric relaxation processes originate in the permanent dipole moment in the substance.

In an actual measurement, using a set of metal electrodes forming a capacitor, the complex permittivity also contains effects of the electrode polarization and the dc conductivity in addition to these processes with molecular origin especially in the low frequency region such as less than $10^{-3} \mathrm{~Hz}$. In this case, the complex permittivity, $\varepsilon^{*}(\omega)=\varepsilon^{\prime}(\omega)-i \varepsilon^{\prime \prime}(\omega)$, is expressed as follows:

$$
\varepsilon^{*}(\omega)-\varepsilon_{\infty}=\sum F_{i}^{*}(\omega)+F_{\text {elec }}^{*}(\omega)+F_{\sigma}^{*}(\omega) .
$$

The function $F_{i}^{*}(\omega)$ describes the dielectric relaxation processes with molecular origin such as the $\alpha$ process, the slow $\beta$ process, the fast $\beta$ process, boson peak and so on. $F_{\text {elec }}^{*}(\omega)$ and $F_{\sigma}^{*}(\omega)$ represent the electrode polarization and the dc conductivity, respectively. Large electrode polarization and dc conductivity bring difficulty to the dielectric measurement for investigation of the slower dynamics. Even removing impurities can reduce the conductive processes, it is usually impossible to erase the effect completely. Therefore, we need to evaluate these effects of $F_{\text {elec }}^{*}(\omega)$ and $F_{\sigma}^{*}(\omega)$ precisely to study the behavior of $\Sigma F_{i}^{*}(\omega)$ in Eq. (1). However, such evaluation has been difficult in the complex permittivity measurement of glass-forming liquids especially with hydrogen bonding networks mainly due to technical limitation. Finally, dielectric study at frequencies lower than the $\alpha$ relaxation frequency has not been performed very much so far.

In this study, we performed the complex permittivity measurement for the glass-forming liquids (sorbitol, xylitol, glycerol and sorbitol-xylitol mixtures) in the frequency range between $10 \mu \mathrm{Hz}$ and $500 \mathrm{MHz}$ even at temperatures where the relaxation frequency of the $\alpha$ process is very large. We have found a dielectric relaxation process (ultraslow process, USP) with the relaxation time much larger than that of the $\alpha$ process [7]. The relaxation frequency depends on the molecular size systematically and shows non-Arrhenius temperature dependence. The relaxation strength of the USP decreases with time. On the basis of these experiments, we will consider the origin of the USP.

\section{EXPERIMENT}

Crystal powders of xylitol $\left(\mathrm{C}_{5} \mathrm{H}_{12} \mathrm{O}_{5}\right)$, sorbitol $\left(\mathrm{C}_{6} \mathrm{H}_{14} \mathrm{O}_{6}\right)$ and absolute liquid glycerol $\left(\mathrm{C}_{3} \mathrm{H}_{8} \mathrm{O}_{3}\right)$ were purchased from Kishida Chemical. In the case of pure sorbitol and xylitol, the samples of powder were annealed at the temperature near the melting temperature for $24 \mathrm{~h}$ in a vacuum chamber to remove the impurities, especially moisture. In the case of sorbitol-xylitol mixtures, powder of sorbitol and xylitol were mixed before the annealing. We prepared three samples for the mixtures with different mole fraction of xylitol, $x(x$ $=0.3,0.5,0.7)$. In the case of glycerol, we used absolute glycerol, without further purification. All the samples pre- 
pared in a way just described above were quenched to the temperature for the measurement in the dielectric cell.

We have measured the complex permittvity of sorbitol, xylitol and the mixtures in the frequency range between $10 \mu \mathrm{Hz}$ and $500 \mathrm{MHz}$ at temperatures between 250 and $346 \mathrm{~K}$, and that of glycerol from $1 \mathrm{~Hz}$ to $1 \mathrm{MHz}$ at 258-299 K. In order to cover the wide frequency range, four different measuring systems were used. From $10 \mu \mathrm{Hz}$ to $0.3 \mathrm{~Hz}$, a time-domain spectrometer was employed. A step-like voltage, which is produced by a computer with digital-analog converter and a high-speed power amplifier (NF2010), is applied to a three-terminal parallel-plate capacitor filled with the sample. Corresponding charging and discharging currents were measured by an electrometer (KEITHLEY 6521). The complex permittivity is obtained from the Fourier transform of the voltage and the current data with time. From 0.1 to $100 \mathrm{~Hz}$, a fast Fourier transform analyzer (HP35670A) and a current amplifier (KEITHLEY 428) were employed using the same parallel-plate sample cell. We used a $L C R$ meter (HP4284A) and the same parallelplate sample cell as a four-terminal circuit between $20 \mathrm{~Hz}$ and $1 \mathrm{MHz}$. From $1 \mathrm{MHz}$ to $500 \mathrm{MHz}$, a network analyzer (HP4195A) was used with the transmission line method. A coaxial sample cell is located at the end of a coaxial line. The values of the complex permittivity were obtained from reflection measurements. Below $1 \mathrm{MHz}$, the cryostat with the sample cell is set in a bath filled with mixture of water and ethylene glycol. Temperature of the cell was controlled within $0.05 \mathrm{~K}$. Above $1 \mathrm{MHz}$, the temperature of the cell inserted into water jacket was controlled within $0.1 \mathrm{~K}$.

\section{RESULTS}

\section{A. Glycerol}

Complex permittivity of glycerol at $279 \mathrm{~K}$ is shown in Figs. 1(a) and 1(b), respectively. These figures indicate that the low frequency side of the $\alpha$ process is observed in the frequency range between $10 \mathrm{kHz}$ and $1 \mathrm{MHz}$. On the other hand, the contribution of the dc conductivity generally emerges in only the imaginary part of the complex permittivity and is expressed by

$$
F_{\sigma}^{*}(\omega)=\frac{\sigma}{i \omega} .
$$

It is clear that Fig. 1(b) shows large effect of the dc conductivity in the low frequency region. The contribution is evaluated in terms of Eq. (2) as is shown in the same figure with dashed line. Finally, in Fig. 1(c), plots of $\varepsilon^{\prime \prime}(\omega)$ are shown after subtraction of the components of $F_{\sigma}^{*}(\omega)$. Judging by Fig. 1(c), there are three relaxation processes in the measured frequency range and these are assigned to the electrode polarization $\left[F_{\text {elec }}^{*}(\omega)\right]$, the $\alpha$ process $\left[F_{\alpha}^{*}(\omega)\right]$ and a new process $\left[F_{U S P}^{*}(\omega)\right]$. We call the new relaxation process the ultraslow process (USP). It has been reported that high frequency wing (excess wing [8]) is observed on glycerol in the higher frequency range than the $\alpha$ process. Within our experimental frequency range, excess wing cannot be seen and only the $\alpha$ process and the new process were evaluated
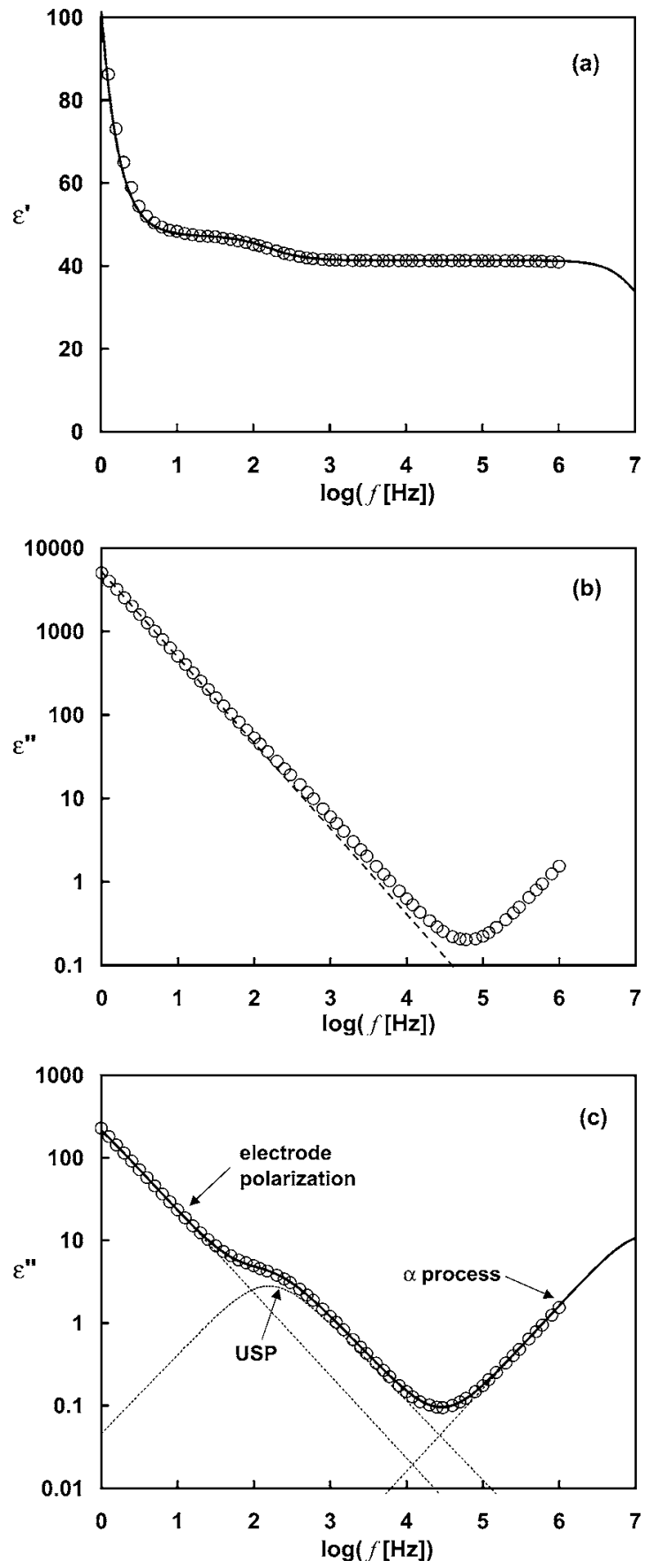

FIG. 1. (a) $\varepsilon^{\prime}(\omega)$, (b) $\varepsilon^{\prime \prime}(\omega)$ of glycerol at $279 \mathrm{~K}$ and (c) $\varepsilon^{\prime \prime}(\omega)$ without the contribution of the dc conductivity. Solid curves in Figs. 1(a) and 1(c) are given by Eq. (3). Dashed line in Fig. 1(b) represents the contribution of the dc conductivity.

as $F_{\alpha}^{*}(\omega)$ and $F_{U S P}^{*}(\omega)$. In this case, we applied the DavidsonCole function [9] for the structural $\alpha$ process $\left[F_{\alpha}^{*}(\omega)\right][8]$ and the Cole-Cole function [10] for the new process $\left[F_{U S P}^{*}(\omega)\right]$. The electrode polarization $\left[F_{\text {elec }}^{*}(\omega)\right]$ is expressed by the high frequency slope of the Davidson-Cole function. We performed a best fitting procedure and the complex permittivity of glycerol at $274 \mathrm{~K}$ can be described very well with the three components. The plots of $\varepsilon^{\prime}(\omega)$ and $\varepsilon^{\prime \prime}(\omega)$ in Figs. 1(a) and 1(c) satisfy the Kramers-Kronig relationships. Therefore, 
the complex permittivity of glycerol, whose contribution of dc conductivity is removed, can be described in terms of Eq. (3)

$$
\begin{gathered}
\varepsilon^{*}(\omega)-\varepsilon_{\infty}=F_{\alpha}^{*}(\omega)+F_{U S P}^{*}(\omega)+F_{\text {elec }}^{*}(\omega), \\
F_{\alpha}^{*}(\omega)=\frac{\Delta \varepsilon_{\alpha}}{\left(1+i \omega \tau_{\alpha}\right)^{\gamma_{\alpha}}}, \\
F_{U S P}^{*}(\omega)=\frac{\Delta \varepsilon_{U S P}}{1+\left(i \omega \tau_{U S P}\right)^{\delta_{U S P}}}, \\
F_{\text {elec }}^{*}(\omega)=\frac{\Delta \varepsilon_{\text {elec }}}{\left(1+i \omega \tau_{\text {elec }}\right)^{\gamma_{\text {elec }}}} .
\end{gathered}
$$

Parameter $\Delta \varepsilon$ is the relaxation strength, $\tau$ is the relaxation time, and $\gamma$ and $\delta$ are the shape parameters that represent asymmetric and symmetric broadenings of relaxation time; $\varepsilon_{\infty}$ is the high frequency limiting permittivity and $\omega$ is an angular frequency. Equation (3) can also express the complex permittivity of glycerol at all the temperatures.

\section{B. Sorbitol, xylitol and their mixtures}

Complex permittivity of sorbitol-xylitol mixture $(x=0.7)$ at $260 \mathrm{~K}$ is shown in Figs. 2(a) and 2(b), respectively. The contribution of the de conductivity is not observed with a time-domain spectrometer from $10 \mu \mathrm{Hz}$ to $0.3 \mathrm{~Hz}$ [11]. The contribution of the electrode polarization is negligible for this system at this temperature. As we have already reported [3-6], the $\alpha$ process can be described well by using the Havriliak-Negami function [12] and the slow $\beta$ process by Cole-Cole function on sorbitol-xylitol mixtures. It is clear that the $\alpha$ process and the slow $\beta$ process are observed in Figs. 2(a) and 2(b). In addition to these processes, there is another relaxation process at the lower frequency range of the $\alpha$ process. Thus, we again chose the Cole-Cole function for the USP as we did for the glycerol. In this case, the corresponding formula for Eq. (1) without $F_{\text {elec }}^{*}(\omega)$ and $F_{\sigma}^{*}(\omega)$ is given by

$$
\begin{gathered}
\varepsilon^{*}(\omega)-\varepsilon_{\infty}=F_{\alpha}^{*}(\omega)+F_{\beta}^{*}(\omega)+F_{U S P}^{*}(\omega) . \\
F_{\alpha}^{*}(\omega)=\frac{\Delta \varepsilon_{\alpha}}{\left\{1+\left(i \omega \tau_{\alpha}\right)^{\delta_{\alpha} \gamma_{\gamma}}\right.} . \\
F_{\beta}^{*}(\omega)=\frac{\Delta \varepsilon_{\beta}}{1+\left(i \omega \tau_{\beta}\right)^{\delta_{\beta}}} .
\end{gathered}
$$

The parameter $\gamma$ and $\delta$ in Havriliak-Negami function are shape parameters that represent asymmetric and symmetric broadening of relaxation time. The complex permittivity of all the samples on sorbitol-xylitol mixtures can be described very well with Eq. (7).

\section{Relaxation frequency}

Figure 3 shows the Arrhenius diagram of the USP for all the materials. The relaxation frequency $f_{m}$ is plotted against
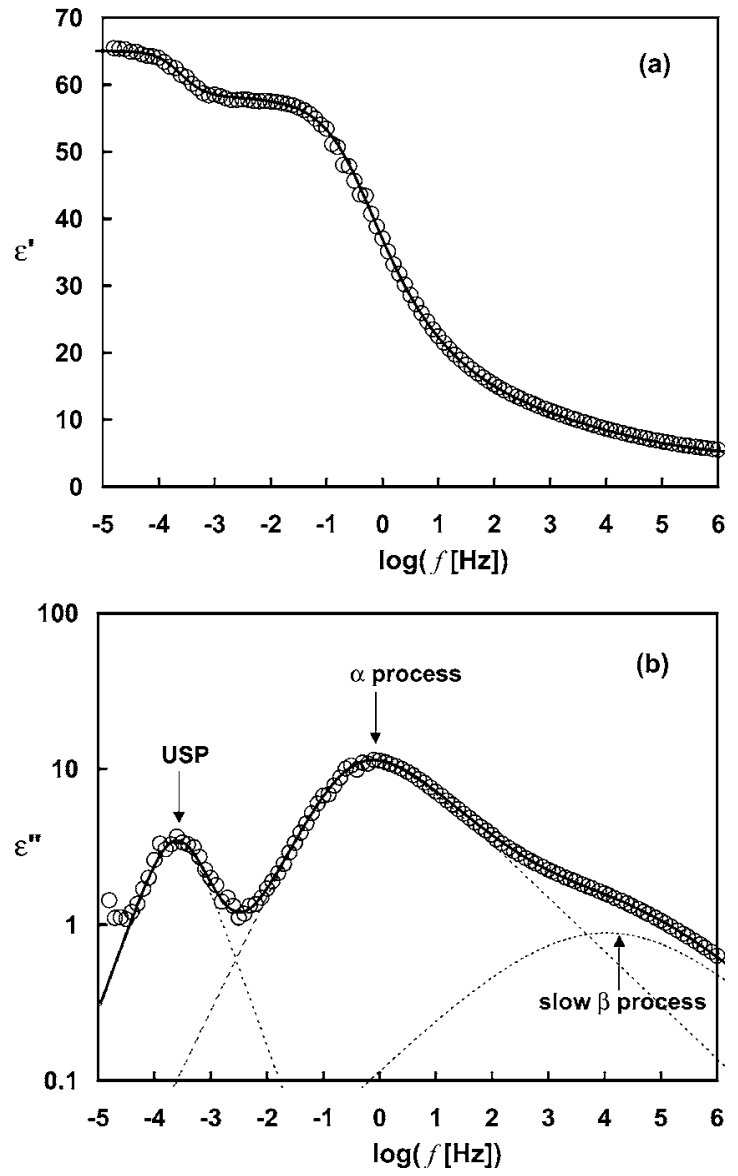

FIG. 2. (a) $\varepsilon^{\prime}(\omega)$ and (b) $\varepsilon^{\prime \prime}(\omega)$ of sorbitol-xylitol mixture $(x=0.7)$ at $260 \mathrm{~K}$. Solid curves are given by Eq. (7).

reciprocal temperature. It is clear that the relaxation frequency shows non-Arrhenius temperature dependence from the diagram. It should be pointed that the loci for the USP depend on molecular weight systematically. Figure 4 shows the relation between the USP and other processes (the $\alpha$

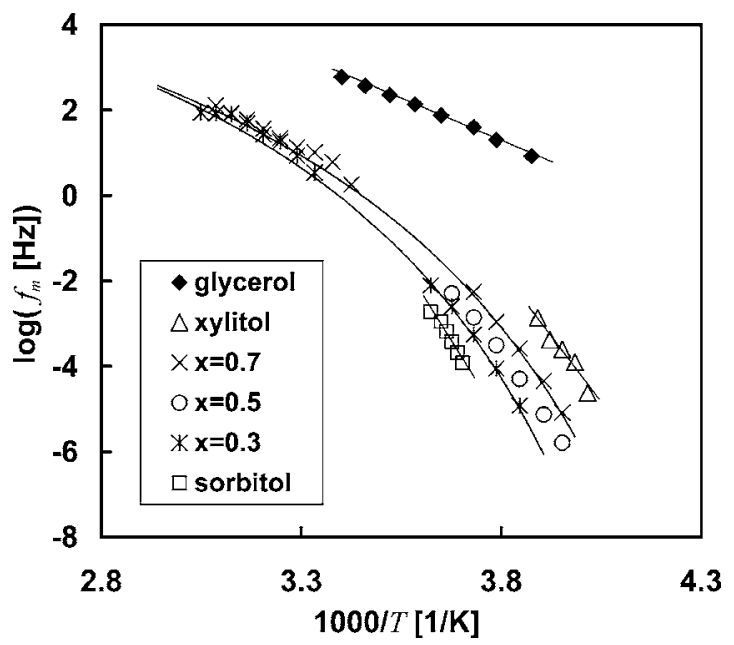

FIG. 3. Arrhenius diagram of the USP in all the samples. Solid curves are just guide for eyes. 


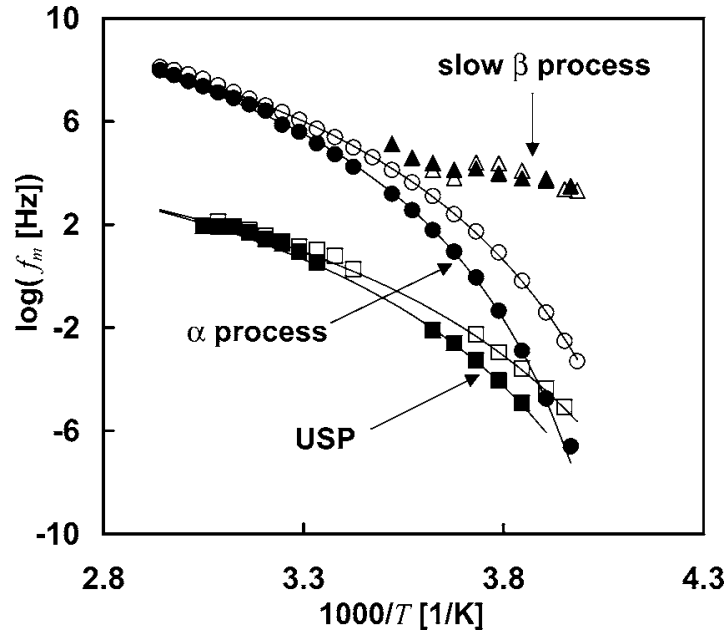

FIG. 4. Arrhenius diagram of sorbitol-xylitol mixtures $[x=0.3$ (solid symbols) and $x=0.7$ (open symbols)]. Solid curves are just guide for eyes.

process and the slow $\beta$ process) on sorbitol-xylitol mixtures $(x=0.3,0.7)$. This figure indicates the USP approaches the $\alpha$ process with decreasing the temperature.

\section{Time dependence of the ultraslow process}

Although the experimented values of the relaxation time are reproducible for each measurement, we could not get good reproducibility for $\Delta \varepsilon$. The most worse case was for glycerol. Therefore, we measured the time dependence of the USP on glycerol at 264, 274, and 293 K. Figures 5(a) and 5(b) show the time dependence of the complex permittivity at $274 \mathrm{~K}$. The contribution of the dc conductivity is removed in Fig. 5(b). The initial time was chosen to be the time when the first measurement was made. It is clear from these figures that $\Delta \varepsilon$ of the USP decreases with time. The dielectric relaxation parameter of this measurement was obtained by the same procedure as we already explained in the previous section. According to this result, we conformed that the relaxation frequency $f_{m}$ does not change with time. Figures 6 and 7 show the behavior of $\Delta \varepsilon(t) / \Delta \varepsilon_{\text {initial }}$ and symmetric shape parameter $\delta$ with time. In Fig. 6, we employed an exponential function (10) to estimate the rate of decrease of $\Delta \varepsilon$ in time

$$
\Delta \varepsilon(t)=A \exp \left(-\frac{t}{\tau_{\text {relax }}}\right) .
$$

It was found that this function works very well only for the short time region as is indicated in Fig. 6. Therefore, we can roughly estimate the rate of decrease, $\tau_{\text {relax }}$. The estimation indicates that $\tau_{\text {relax }}$ is the order for 10000 seconds and higher temperature gives smaller value of $\tau_{\text {relax }}$. Figure 6 also shows the deviation, in the long time region, of $\Delta \varepsilon(t)$ from the function (10) determined by using data in short time region.

\section{DISCUSSION}

According to the fact that $\Delta \varepsilon(t)$ decreases with time, the USP is considered to be essentially a nonequilibrium phe-
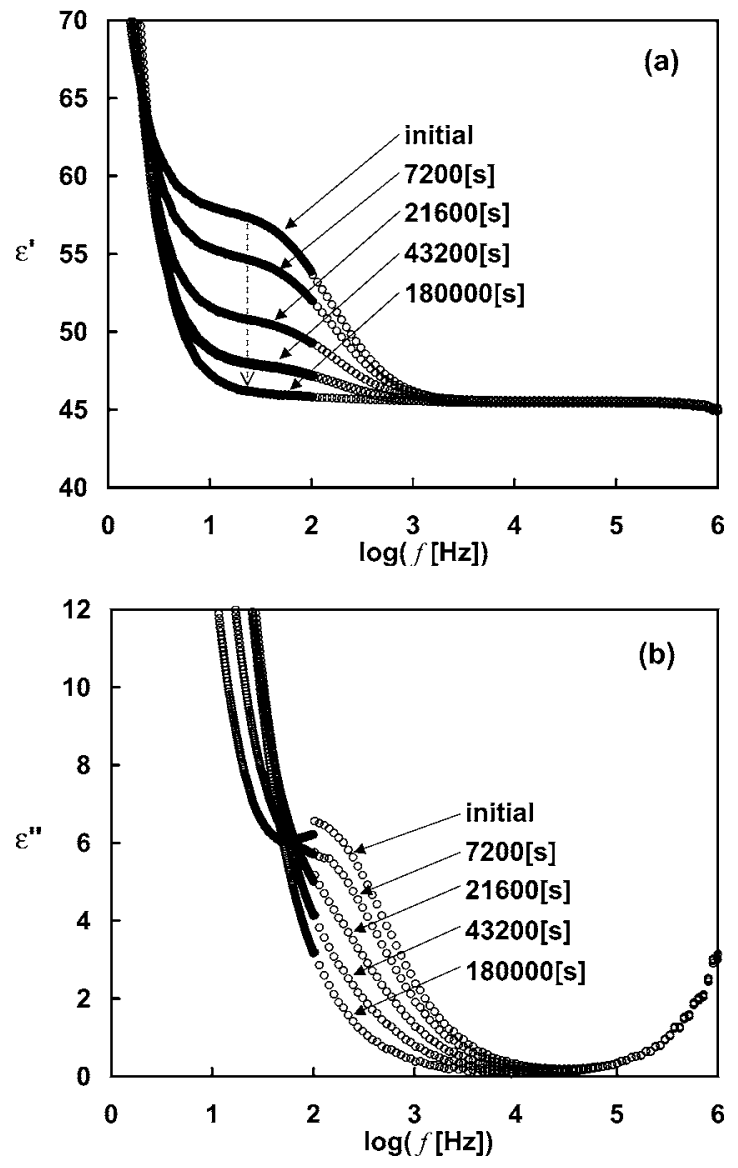

FIG. 5. Time dependence of $\varepsilon^{\prime}(\omega)$ and $\varepsilon^{\prime \prime}(\omega)$ of glycerol at $274 \mathrm{~K}$. The values of $\varepsilon^{\prime \prime}(\omega)$ do not contain the effect of the dc conductivity.

nomenon. The relaxation frequency of the USP depends on the molecular weight systematically. And temperature dependence of the relaxation frequency $f_{m}$ of the USP follows a non-Arrhenius manner. Moreover, temperature dependence of $f_{m}$ of the mixtures shows intermediate behavior between

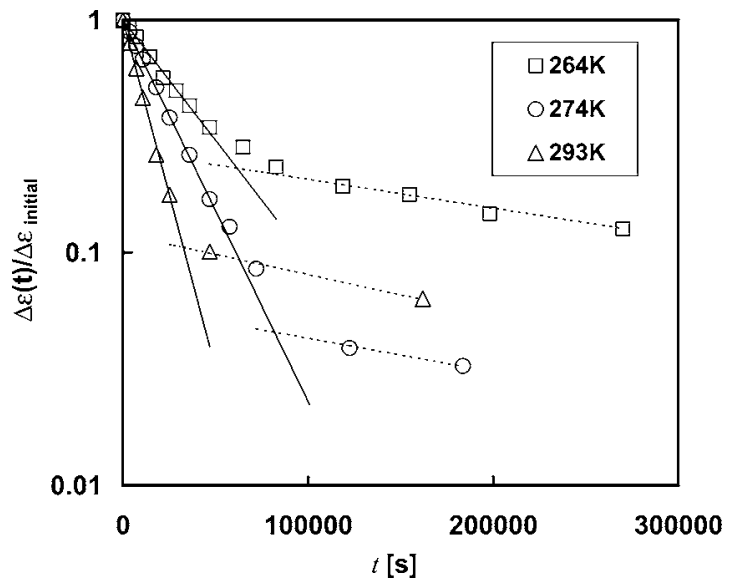

FIG. 6. Time dependence of $\Delta \varepsilon(t) / \Delta \varepsilon_{\text {initial }}$ for glycerol at 264, 274, and $293 \mathrm{~K}$. Solid line represents the best fit results of Eq. (10) for $\Delta \varepsilon(t) / \Delta \varepsilon_{\text {initial }}$ using data in the short time range. The dotted line describes the deviation of long time behavior of $\Delta \varepsilon(t) / \Delta \varepsilon_{\text {initial }}$ from that of short time behavior. 


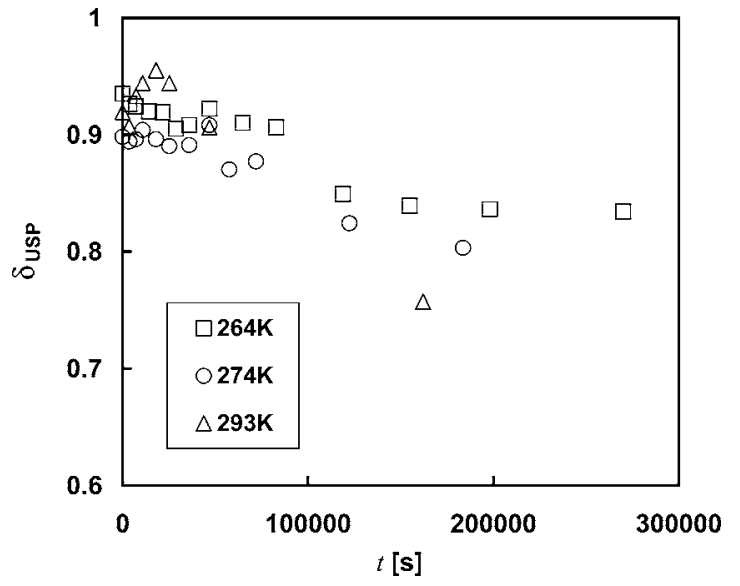

FIG. 7. Time dependence of symmetric shape parameter $\delta_{U S P}$ in Eq. (5) for glycerol at 264, 274, and $293 \mathrm{~K}$.

sorbitol and xylitol as is indicated in Fig. 3. These features of the USP are very similar to that of the $\alpha$ process.

Recently, Papadopoulos et al. [13] have reported a dielectric relaxation process with the relaxation time larger than that of the structural $\alpha$ process on poly(2-vinylpyridine). The relaxation time of the process is slower than that of the $\alpha$ process by three orders and depends on the molecular weight of the polymer systematically. The loci of the process follow a Vogel-Fulcher-Tammann [14-16] manner and have the same $T_{0}$ parameter, which is the ideal glass temperature, as that of the $\alpha$ process. In our measurement, we have not analyzed the loci of the USP in detail because of the limitation in the frequency range due to the experimental difficulties. However, the basic features of the slower process are very similar to those for the USP.

Even the slower process reported on P2VP is similar to the USP on polyhydric alcohols in our study, time dependence of the relaxation strength has not been reported on the P2VP, unlike glycerol. According to our experiments on the sorbitol and xylitol, the time dependence of the relaxation strength could not be observed clearly. This situation indicates that the relaxation strength of the USP for larger molecules does not change very much with time or the rate of the change is too slow to be observed in our experimental time scale. This consideration is consistent with the fact that such time dependence is not observed for polymeric materials.

Fischer and his co-workers have reported a slow mode in the low molecular weight and polymeric glass-forming liquids from their scattering measurements [17-24]. The intensity of light and x-ray scattering in the small $q$ region is much higher than the value expected for a simple liquid and the excess intensity is caused by long-range density fluctuation. According to static light scattering measurements the correlation lengths are ten to several hundreds nanometers, which are much longer than the length corresponding to the $\alpha$ process being suggested from recent works [25-27]. This fact is consistent with the behavior of the USP. They explained the long-range density fluctuation with the spatial heterogeneities in liquid. It is very important to study the relations between Fischer's concept and the origin of the USP.

\section{CONCLUSION}

Another process, the ultraslow process (USP), in addition to the structural $\alpha$ process and the slow $\beta$ process, was observed in supercooled polyhydric alcohol systems. The USP emerges at much lower frequency range than the $\alpha$ process. The relaxation time of the USP shows non-Arrhenius temperature dependence and depends on the molecular size systematically. The USP approaches the $\alpha$ process with decreasing temperature. On glycerol, the dielectric relaxation strength decreases exponentially with time and the decreasing rate changes according to the temperatures for the early time region. The rate of decrease deviates halfway from the exponential function. Another exponential function is applied to the late time region. The order of the decreasing rate changes from 10000 to $100000 \mathrm{~s}$ when the relaxation strength becomes small, around 1.0.
[1] F. Kremer and A. Schonhals, Broadband Dielectric Spectroscopy (Springer, Berlin, 2003).

[2] G. P. Johari and M. Goldstein, J. Chem. Phys. 53, 2372 (1970).

[3] R. Nozaki, D. Suzuki, S. Ozawa, and Y. Shiozaki, J. NonCryst. Solids 235, 393 (1998).

[4] R. Nozaki, S. Ozawa, and Y. Shiozaki, in Slow Dynamics in Complex Systems, edited by M. Tokuyama and I. Oppenheim, AIP Conf. Proc. No. 469 (AIP, Melville, New York, 1999), p. 521.

[5] A. Minoguchi and R. Nozaki, J. Non-Cryst. Solids 307, 246 (2002).

[6] A. Minoguchi, K. Kei, and R. Nozaki, Phys. Rev. E 68, 031501 (2003).

[7] Y. Yomogida, A. Minoguchi, and R. Nozaki, in Slow Dynamics in Complex Systems, edited by M. Tokuyama and I. Oppenheim, AIP Conf. Proc. No. 708 (AIP, Melville, New York,
2004), p. 667.

[8] P. Lunkenheimer, R. Wehn, T. Riegger, and A. Loidl, J. NonCryst. Solids 307, 336 (2002).

[9] D. W. Davidson and R. H. Cole, J. Chem. Phys. 18, 1417 (1951).

[10] K. S. Cole and R. H. Cole, J. Chem. Phys. 9, 341 (1941).

[11] N. G. McCrum, B. E. Read, and G. Williams, Anelastic and Dielectric Effects in Polymeric Solids (Dover, New York, 1991), pp. 214-219.

[12] S. Havriliak and S. Negami, Polymer 8, 161 (1967).

[13] P. Papadopoulos, D. Peristeraki, G. Floudas, G. Koutalas, and N. Hadjichristidis, Macromolecules 37, 8116 (2004).

[14] H. Vogel, Phys. Z. 22, 645 (1921).

[15] G. S. Fulcher, J. Am. Ceram. Soc. 8, 339 (1925).

[16] G. Tammann and W. Hesse, Z. Anorg. Allg. Chem. 156, 245 (1926).

[17] B. Gerharz, G. Meier, and E. W. Fischer, J. Chem. Phys. 92, 
$1770(1990)$.

[18] E. W. Fischer, G. Meier, T. Rabenau, A. Patkowski, W. Steffen, and W. Thonnes, J. Non-Cryst. Solids 131, 134 (1991).

[19] E. W. Fischer, Physica A 201, 183 (1993).

[20] T. Kanaya, A. Patkowski, E. W. Fischer, J. Seils, H. Glaser, and K. Kaji, Acta Polym. 45, 137 (1994).

[21] T. Kanaya, A. Patkowski, E. W. Fischer, J. Seils, H. Glaser, and K. Kaji, Macromolecules 28, 7831 (1995).

[22] A. Patkowski, Th. Thurn-Albrecht, E. Banachowicz, W. Steffen, P. Bösecke, T. Narayanan, and E. Fischer, Phys. Rev. E 61, 6909 (2000).
[23] A. Patkowski, E. W. Fischer W. Steffen, H. Glaser, M. Baumann, T. Ruths, and G. Meier, Phys. Rev. E 63, 061503 (2001).

[24] M. Wind, R. Graf, A. Heuer, and H. W. Spiess, Phys. Rev. Lett. 91, 155702 (2003).

[25] E. Donth, Z. Phys. Chem. (Leipzig) 258, 977 (1977).

[26] E. W. Fischer, E. Donth, and W. Steffen, Phys. Rev. Lett. 68, 2344 (1992).

[27] E. W. Fischer and A. Zetsche, Polym. Prepr. (Am. Chem. Soc. Div. Polym. Chem.) 33, 78 (1992). 